\title{
Phage Abp1 Rescues Human Cells and Mice from Infection by Pan-Drug Resistant Acinetobacter Baumannii
}

\author{
Supeng Yin ${ }^{a}$ Guangtao Huang ${ }^{a}$ Yulong Zhanga, c Bei Jiang ${ }^{a}$ Zichen Yang ${ }^{a}$ \\ Zhiwei Dong ${ }^{\mathrm{a}}$ Bo You ${ }^{\mathrm{a}}$ Zhiqiang Yuan ${ }^{\mathrm{a}}$ Fuquan Hu${ }^{\mathrm{b}}$ Yan Zhao ${ }^{\mathrm{b}}$ Yizhi Peng $^{\mathrm{a}}$ \\ aState Key Laboratory of Trauma, Burns and Combined Injury, Institute of Burn Research, Southwest \\ Hospital, Third Military Medical University, Chongqing, ${ }^{b}$ Department of Microbiology, Third Military \\ Medical University, Chongqing, 'Number 474 Hospital, PLA, China
}

\author{
Key Words \\ A. baumannii $\cdot$ Phage therapy $\cdot$ Local infection - Systemic infection $\cdot$ HeLa cell $\cdot$ THP-1 cell $•$ \\ Cytotoxicity
}

\begin{abstract}
Background/Aims: As an "ESKAPE" pathogen, Acinetobacter baumannii is one of the leading causes of drug-resistant infections in humans. Phage therapy may be a useful strategy in treating infections caused by drug-resistant $A$. baumannii. Among 21 phage strains that were isolated and described earlier, we investigated the therapeutic efficacy of Abp1 because of its relatively wide host range. Methods: Phage stability assays were used to evaluate thermal and $\mathrm{pH}$ stability of Abp1. Abp1 was co-cultured with A. baumannii (AB1) over a range of multiplicities of infection to determine its bactericidal efficacy. HeLa or THP-1 cells were used in the cytotoxicity and protection assays. Finally, the therapeutic effects of Abp1 on local and systemic A. baumannii infection in mice were determined. Results: We found that Abp1 exhibits high thermal and $\mathrm{pH}$ stability and has a low frequency of lysogeny. Bacteriophage resistance also occurs at a very low frequency $\left(3.51 \pm 0.46 \times 10^{-8}\right)$, and Abp1 can lyse almost all host cells at a MOI as low as 0.1 . Abp1 has no detectable cytotoxicity to HeLa or THP-1 cells as determined by LDH release assay. Abp1 can rescue HeLa cells from A. baumannii infection, even if introduced 2 hours post infection. In both local and systemic $A$. baumannii infection mouse models, Abp1 treatment exhibits good therapeutic effects. Conclusion: Abp1 is an excellent candidate for phage therapy against drug-resistant $A$. baumannii infections.
\end{abstract}

\section{Introduction}

Acinetobacter baumannii (A. baumannii) is a non-fermentative, non-motile, catalasepositive, Gram-negative bacterium [1,2], widely found in soil, water, sewage, and many healthcare environments. In clinical settings, this pathogen causes severe pneumonia,

Yizhi Peng

and Yan Zhao 
as well as infections in the urinary tract, bloodstream, and wounds [2-4]. In the 1970s, $A$. baumannii was susceptible to most antibiotics. However, an increasing number of clinical $A$. baumannii strains now exhibit multi-drug and pan-drug resistance. The rapid development of antibiotic resistance is due to the acquisition of plasmids, transposons, and integrons that carry clusters of genes encoding resistance to several antibiotic families [2, 4]. Effective management of $A$. baumannii infections has become an increasingly urgent issue, requiring the development of new tools.

A growing body of literature has validated the use of phages for therapy and prophylaxis in the war against drug-resistant bacteria [5-9], and many lytic phages have been isolated and tested in animals for their efficacy against bacterial infections. In 2011, the U.S. Food and Drug Administration approved a mixture of bacteriophages as a food additive for protection against foodborne bacterial disease. In addition, promising results have been obtained using phages isolated on-demand to eradicate gram-negative bacteria [10].

In a previous report we described $A b p 1$, a bacteriophage isolated from hospital sewage [11]. Abp1 is a lytic phage of the pan-drug resistant $A$. baumannii strain AB1. In this study, we analyzed the potential of Abp 1 as a phage therapy candidate and tested its effects in both local and systemic mouse infection models.

\section{Materials and Methods}

\section{Bacterial strain and phages}

A. baumannii AB1 was isolated from a burn patient at Southwest Hospital in Chongqing, China. It is resistant to multiple antibiotics, including aminoglycosides, carbapenems, cephalosporins, tetracyclines, and quinolones, but is sensitive to polymyxin with a MIC of $0.236 \mathrm{mg} / \mathrm{L}$ [11]. Abp1 is a lytic phage of AB1, isolated from sewage at the same location. The characteristics of this phage are described in our previous report [11]. Endotoxin was removed from Abp1 cultures using the ToxinEraser endotoxin removal kit (GenScript, China).

\section{Bacteriophage resistance assay}

Serial dilutions of $A$. baumannii $\mathrm{AB} 1$, ranging from 10 to $10^{9} \mathrm{CFU} / \mathrm{ml}$, were mixed with $5 \times 10^{10}$ plaqueforming units (PFU) of $\mathrm{Abp} 1.10 \mu \mathrm{L}$ from each infected culture was spotted onto an agar plate and incubated at $37^{\circ} \mathrm{C}$ for $24 \mathrm{~h}$. Colonies formed by surviving bacteria were considered to be resistant to Abp1. Bacteriophage resistance was calculated as the ratio of CFU relative to the number of plated bacteria.

\section{Thermal and $p H$ stability of Abp1}

To test the thermal stability of Abp1, phage samples $\left(7.5 \times 10^{10} \mathrm{PFU}\right.$ in a volume of $\left.1.5 \mathrm{ml}\right)$ were incubated at $50,60,70$, and $80^{\circ} \mathrm{C} .100 \mu \mathrm{L}$ aliquots were withdrawn after $5 \mathrm{~min}, 15 \mathrm{~min}, 40 \mathrm{~min}$, and $1 \mathrm{~h}$, and diluted 10fold. $10 \mu \mathrm{l}$ of diluted phage was combined with $200 \mu \mathrm{l}$ host bacteria, incubated for 15 minutes, mixed with 3 $\mathrm{ml}$ of $0.75 \% \mathrm{LB}$ agar, and poured onto plates containing solid medium to determine phage titer. For the $\mathrm{pH}$ stability assay, aliquots were collected one hour after incubation in liquid media ranging from pH 2 to 13.

\section{Cytotoxicity of Abp1 to HeLa and THP-1 cells}

LDH release from HeLa and THP-1 cells was determined using a CytoTox96 cytotoxicity assay (Promega G1780) [12]. HeLa cells were cultured in Dulbecco's Modified Eagle's Medium and THP-1 cells in Roswell Park Memorial Institute 1640 Medium, both supplemented with fetal bovine serum (10\%) and penicillin- streptomycin (1\%), and incubated in $5 \% \mathrm{CO}_{2}$ at $37^{\circ} \mathrm{C}$. When cells reached $70-80 \%$ confluence, various amounts of Abp1 were added to the wells. 2\% Triton X-100 was added as a positive control, and an equal volume of PBS was used as a negative control. After incubation for 24 hours, $50 \mu \mathrm{L}$ of supernatant was added to the wells of a 96-well plate containing $50 \mu \mathrm{L}$ of reaction mixture. The plate was incubated at room temperature for 30 minutes, and then $50 \mu \mathrm{L}$ Stop Solution was added to halt the reaction. Absorbance was measured at $490 \mathrm{~nm}$.

\section{KARGER}




\section{Cellular Physiology Cell Physiol Biochem 2017;44:2337-2345 \begin{tabular}{l|l} 
and Biochemistry Pul: 10.1159/000486117 & $\begin{array}{l}\text { (c) } 2017 \text { The Author(s). Published by S. Karger AG, Basel } \\
\text { www.karger.com/cpb }\end{array}$
\end{tabular} \\ \begin{tabular}{l|l} 
Published online: December 15, 2017 & www.karger.com/cpb \\
\hline Yin et al: Phage Abp1 in Treating A Baumannii Infections
\end{tabular}}

\section{Killing (lysis) of AB1 by Abp1 infection at various MOI}

To conduct killing assays, an overnight culture of AB1 was diluted 1:100 into $50 \mathrm{ml}$ of medium and incubated for 2 hours at $37^{\circ} \mathrm{C}$ to reach a cell density of approximately $5 \times 10^{7} \mathrm{CFU} / \mathrm{ml}\left(\mathrm{OD}_{600}=0.5\right)$. One milliliter of the culture was added to the wells of a 24-well plate, followed by addition of $100 \mu \mathrm{l}$ diluted Abp1. To achieve multiplicity of infection (MOI) values of $100,10,1$, and 0.1 , a total of $5 \times 10^{9} \mathrm{PFU}, 5 \times 10^{8}$ PFU, $5 \times 10^{7} \mathrm{PFU}$ and $5 \times 10^{6} \mathrm{PFU}$ Abp1 were added to each well. Cell death was analyzed using a microplate reader (SpectraMax M2e, Molecular Devices). The parameters used by SoftMax Pro were as follows: read time interval $=1$ hour, with shaking for 50 minutes at $37^{\circ} \mathrm{C}$ between reads, for a total of 8 hours. Absorbance at $600 \mathrm{~nm}$ was recorded.

\section{HeLa cell infection protection assay}

HeLa cells were cultured as described above in 4 wells of a 6-well plate. When cells reached 70-80\% confluence, AB1 $\left(10^{7} \mathrm{CFU}\right)$ in $100 \mu \mathrm{L}$ DMEM medium was added to 2 wells. For the negative controls, DMEM only was added. After 2 hours, Abp1 ( $\left.10^{8} \mathrm{PFU}\right)$ and an equal volume of PBS were added to all wells. The cells were incubated for additional 22 hours, and the number of living cells was determined using trypan blue.

\section{Phage therapy in a mouse local infection model}

The ability of Abp1 to confer protection against AB1 was evaluated using a mouse wound infection model as described previously [13]. Briefly, 36 six-week-old male BALB/c mice were randomly assigned to three groups ( $\mathrm{n}=12$ per group). Each mouse received cyclophosphamide $(150 \mathrm{mg} / \mathrm{kg}$ body weight) via intraperitoneal injection on day -4 and $100 \mathrm{mg} / \mathrm{kg}$ on day -1 . One day after the second injection, mice were anesthetized with amobarbital sodium and shaved. Two $5.0 \times 5.0 \mathrm{~mm}$ full-thickness wounds were created on the dorsal side of each mouse using a skin biopsy punch. $25 \mu \mathrm{l}$ of PBS containing $5.0 \times 10^{4} \mathrm{AB} 1$ cells were deposited on each wound and the region was air-dried for $5 \mathrm{~min}$.

Four hours after infection, mice were inoculated with phage $\left(5.0 \times 10^{8}\right.$ PFU) in PBS either via a subcutaneous injection or by directly pipetting into the wound. Inoculation with PBS alone was used as a negative control. The phage treatments were repeated once a day for 7 days after infection. On days 1, 3, and 7 , wound sizes were measured and recorded.

\section{Phage therapy in a systemic mouse infection model}

In the mouse systemic infection model, 42 six-week-old male BALB/c mice were randomly assigned to three groups ( $\mathrm{n}=14$ per group). $200 \mu \mathrm{l}$ of PBS containing $5.0 \times 10^{7} \mathrm{AB} 1$ cells was injected intraperitoneally into the mice. Immediately after infection, phage $\left(5.0 \times 10^{8} \mathrm{PFU}\right)$ in PBS were injected intraperitoneally on the same side of the mouse used for the bacterial injection. Phage were injected daily for 6 days after infection. Because AB1 is sensitive to polymyxin $B$, this antibiotic was used as a positive control in the antibiotic treatment group, which received $10 \mathrm{mg} / \mathrm{kg}$ polymyxin B daily for 6 days. The same volume of PBS was injected as a negative control in the third group. The animals were monitored for seven days and the survival rate of each group was calculated. During the observation period, dead mice were dissected immediately to obtain the livers and kidneys. 7 days post-infection, survivors were also sacrificed and dissected. For all animals, livers and kidneys were weighed, homogenized in $1 \mathrm{ml}$ saline, serially diluted, and bacterial counts and phage titers were determined.

\section{Ethics statement}

All animal experiments complied with the International Guiding Principles for Biomedical Research involving Animals (1985) and were approved by the Laboratory Animal Welfare and Ethics Committee of Southwest Hospital, Third Military Medical University.

\section{Statistical analysis}

Data were analyzed by one-way analysis of variance (ANOVA) or log-rank test (Mantel-Cox) as appropriate. A value of $P<0.05$ was considered significant. 


\section{Results}

\section{Characteristics of phage Abp1}

Abp1 was isolated from sewage at Southwest Hospital. It has a genome size of $42 \mathrm{~kb}$ containing 51 genes, including an endolysin gene [14] as expected for a lytic phage. As shown in Fig. 1A, Abp1 can cause lysis in a bacterial culture after 3 hours, and has the ability to form large and transparent plaques (Fig. 1B). We attempted to isolate lysogens of Abp1 by recovery of colonies from the middle of plaques after incubation for one week. However, 10 of the tested colonies were still sensitive to Abp1, suggesting that they are not lysogens. Abp1 is a member of the Podoviridae family, it has a short tail and regular icosahedral head about 60-70 nm diameter. (Fig. 1C). A. baumannii AB1 resistance to Abp1 occurs at a frequency of $3.51 \pm 0.46 \times 10^{-8}$.

\section{Phage stability}

As shown in Fig. 2A, Abp1 is relatively thermostable, with high survival rates after incubation at $50{ }^{\circ} \mathrm{C}$. However, only $0.01 \%$ of phage were viable after $1 \mathrm{~h}$ incubation at $60{ }^{\circ} \mathrm{C}$, after incubation for $15 \mathrm{~min}$ at 70 or $80^{\circ} \mathrm{C}$, almost no phage survive. These results demonstrate that Abp1 retains activity well above $37^{\circ} \mathrm{C}$, which simplifies its storage and delivery. As shown in Fig. 2B, Abp1 also exhibits good pH stability. Abp1 survival was high from $\mathrm{pH} 5.0$ to 10.0, although survival rates decreased significantly between $\mathrm{pH} 4.0$ and 5.0, and from 10.0 to 11.0. No viable phage were detected below 3.0 or above 12.0. The broad range of thermal and $\mathrm{pH}$ stability facilitates the application of Abp1 in phagotherapy.

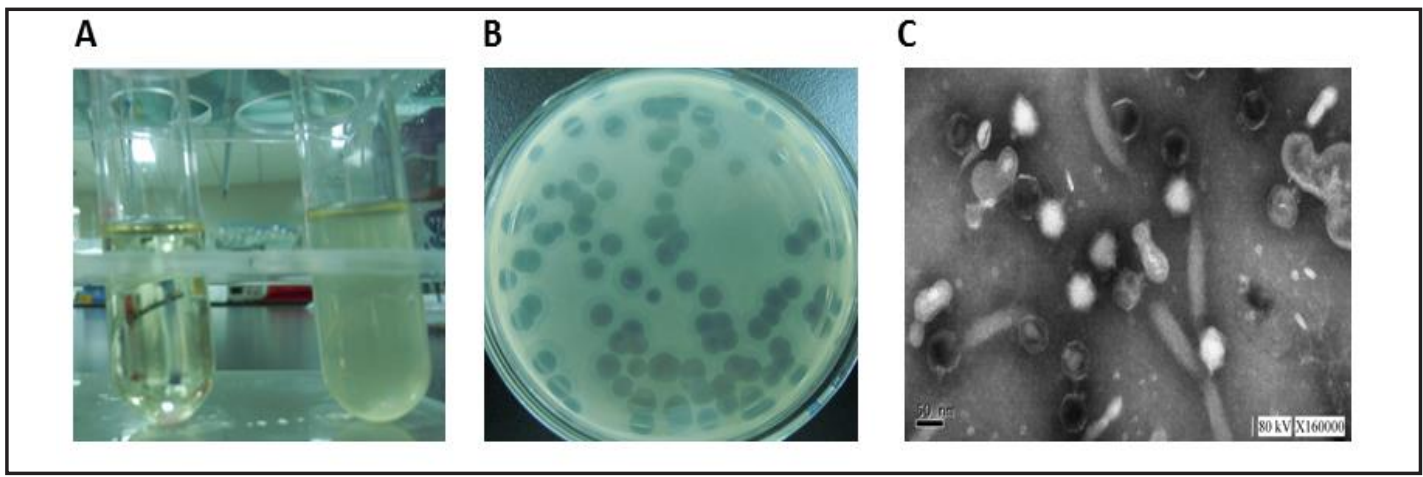

Fig. 1. Characteristics of phage Abp1. (A) Lysis of AB1 by Abp1 in liquid culture. (B) Clear plaques of Abp1 on a lawn of AB1. (C) Electron micrograph of Abp1.

A

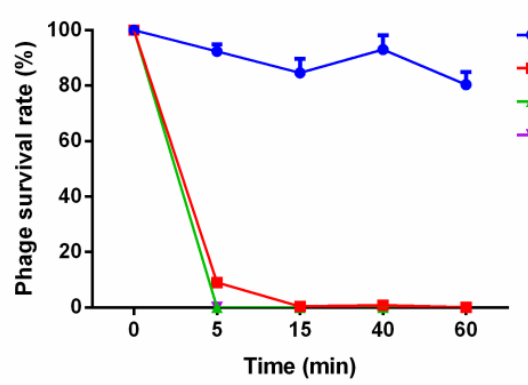

B

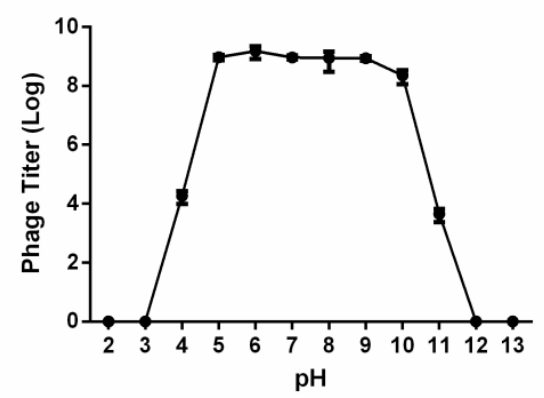

Fig. 2. Phage Abp1 stability. (A) Thermal stability. Phage Abp1 was incubated at various temperatures. Samples were taken at the indicated time points and titered. (B) pH stability. Phage Abp1 was incubated at the indicated $\mathrm{pH}$ conditions for $1 \mathrm{~h}$. Data were obtained from three independent experiments and are shown as mean \pm standard deviation.

\section{KARGER}




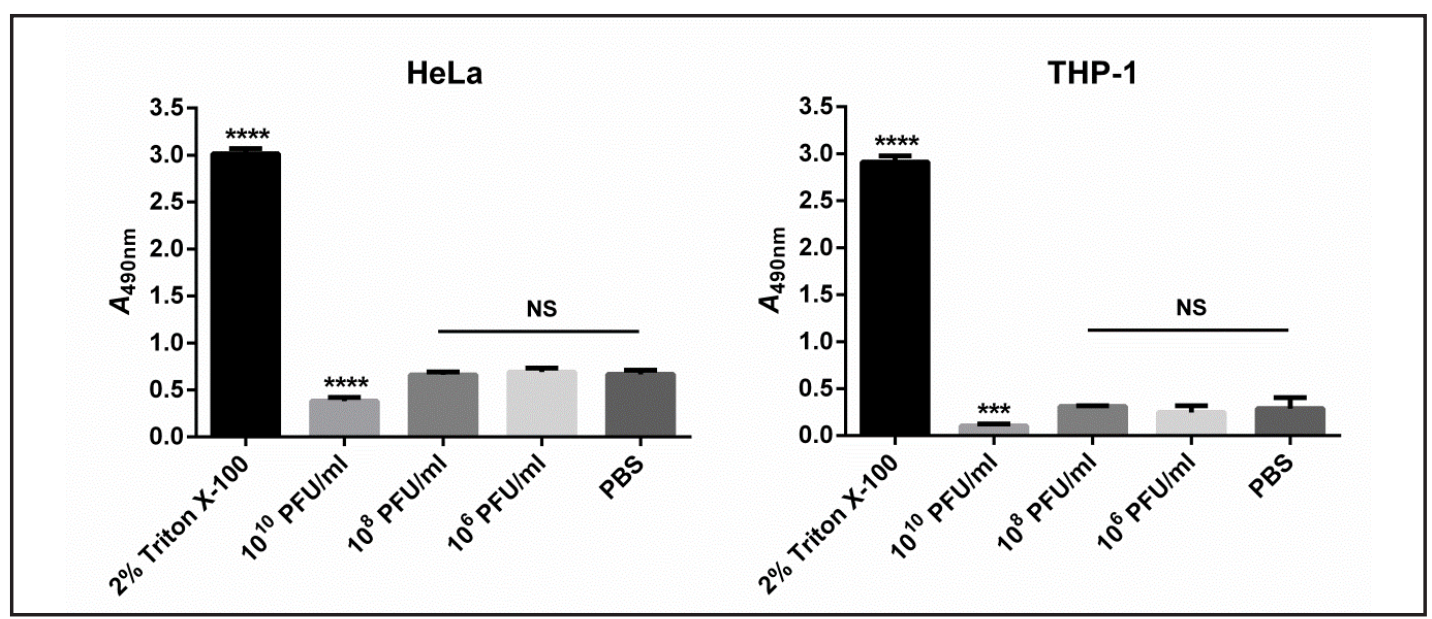

Fig. 3. Cellular cytotoxicity of Abp1. HeLa and THP-1 cells were exposed to various concentrations of Abp1 for 24 hours. Viability (determined using trypan blue) was determined in three independent experiments and is shown as mean \pm standard deviation. ${ }^{* * * *} \mathrm{P}<0.0001$ and ${ }^{* * *} \mathrm{P}<0.001$ indicate significant differences compared with the PBS treated group.

Fig. 4. Bacteriolytic activity of Abp1 at different MOI. Early exponential cultures of $A B 1$ were infected with Abp1 at the indicated MOI. An equal volume of PBS was used as a blank infection control. Data are shown as mean \pm standard deviation from quadruplicate readings in one of three representative experiments.

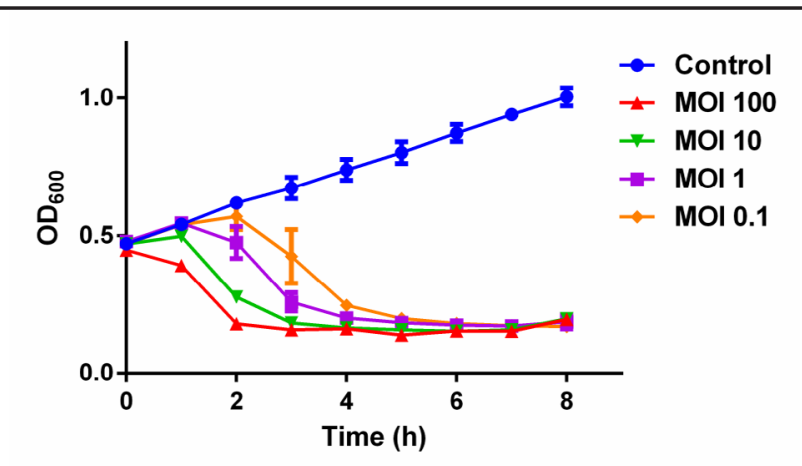

Cytotoxicity of Abp1 to HeLa and THP-1 cells

Potential cytotoxic effects of Abp1 were evaluated using HeLa and THP-1 cell assays (Fig. 3). No cytotoxicity was detected at concentrations of $10^{6}, 10^{8}$, or even $10^{10} \mathrm{PFU} / \mathrm{ml}$, in comparison to the negative PBS group. This suggests that Abp1 is safe for use in antimicrobial treatment in vivo.

\section{Killing of $A B 1$ as a function of Abp1 MOI}

To determine the effective dose range of $\mathrm{Abp} 1, \mathrm{AB} 1$ was infected with phage over a range of MOI, and cultures were monitored for lysis (OD at $600 \mathrm{~nm}$ ). As expected, OD increased steadily during the 8-hour period in the negative control (Fig. 4), but decreased significantly in the cultures infected by Abp1. AB1 growth was inhibited at all MOIs, at the highest phage concentration $(\mathrm{MOI}=100), \mathrm{AB} 1$ failed to grow, and cultures began to decline at an early stage. After slight increases, AB1 growth eventually declined at MOI of 10, 1, and 0.1, with higher phage concentrations resulting in more rapid lysis. Cultures at all four MOI declined to the same $\mathrm{OD}_{600}$ level after 5 hrs incubation.

\section{Protection of HeLa cells against AB1 infection}

After co-incubation with Abp1 for 24 hours, viable HeLa cell counts were identical to those in the negative control (Fig. 5), consistent with the HeLa cell toxicity assay described above. In contrast, the viability of HeLa cells infected with AB1 only for 24 hours decreased significantly. When HeLa cells were treated with phage 2 hours after infection by AB1, their 
Fig. 5. Abp1 rescues HeLa cells after AB1 infection. HeLa cells were pre-infected with $10^{7} \mathrm{CFU}$ AB1 for 2 hours, and then Abp 1 phage $\left(10^{8} \mathrm{PFU}\right)$ were added to the culture medium (AB1+phage). Controls were infected by $A B 1$ but not treated with phage (AB1), uninfected by AB1 but treated with phages (Phage), or both uninfected and untreated (Neg.). Viable cells were counted after incubation for 22 hours. Data are from triplicate experiments. ${ }^{*} \mathrm{P}<0.05$ indicates significant difference between AB1 cells with or without phage treatment.

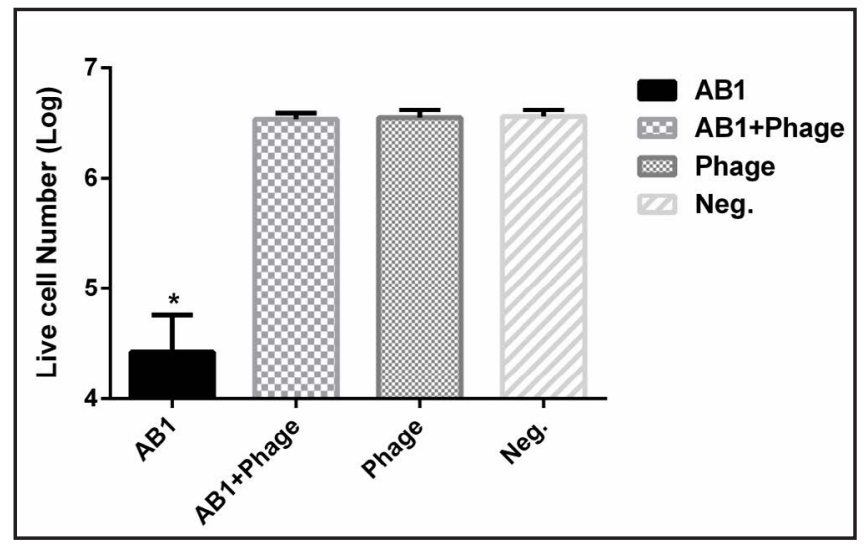

Fig. 6. Wound size in infected mice treated with Abp1. Wound sizes were measured 1,3 , and 7 days after infection and are shown as mean \pm standard deviation. ${ }^{* * *} \mathrm{P}<0.001$ indicates significant difference between the local phage treatment group and the control group.

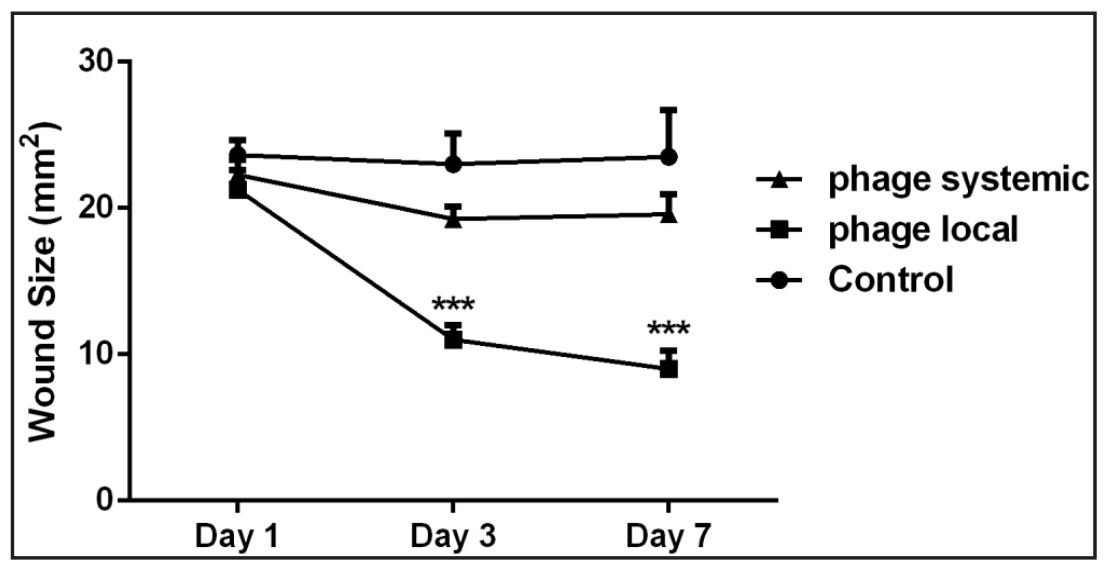

survival was almost identical to the uninfected negative control. This indicates that Abp1 can rescue HeLa cells from AB1 infection, even 2 hours after infection.

\section{Therapeutic application of Abp1 in a local infection}

To investigate the effect of phage therapy on local infection, we devised an A. baumannii infection mouse model. As shown in Fig. 6, 3 and 7 days post-infection, wound sizes in animals receiving locally applied phage were significantly smaller than in mice receiving either systemically administered phage or no treatment (negative control). Although systemic phage therapy was associated with reduced wound size relative to the negative control, the difference was not significant. Interestingly, the wounds in mice receiving locally administered phage had a drier and cleaner appearance than wounds in the other two groups (data not shown). These results suggest that local Abp1 therapy has the potential to be used clinically in the control of local infections by multi-drug resistant $A$. baumannii, and demonstrate that the treatment accelerates wound healing.

\section{Therapeutic application of Abp1 in systemic infection}

In addition to testing the efficacy of Abp1 treatment in local infections, we also tested Abp1 in a systemic AB1 infection model. As shown in Fig. 7A, infected mice receiving no treatment succumbed rapidly. Six mice died 8 hours after infection, with an additional 6 dying during the first day. Only 1 mouse in this group survived the entire seven-day observation period. In contrast, all mice treated with phage or polymyxin B survived the entire 7 days of the observation period. In untreated mice, livers and kidneys contained high bacterial loads, while mice treated with phage or polymyxin B contained almost no bacteria in these organs 
A

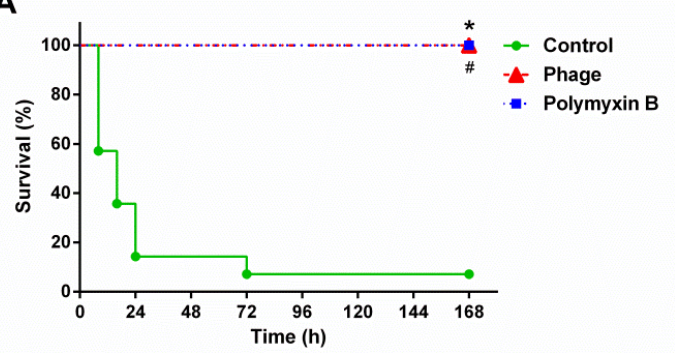

C

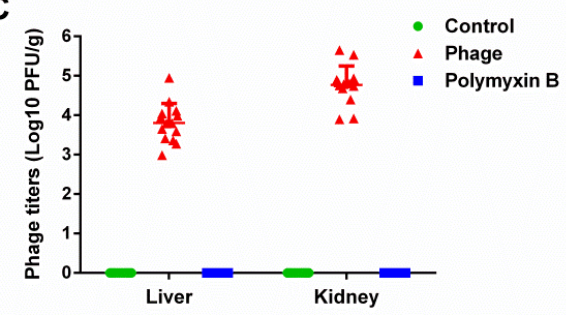

B

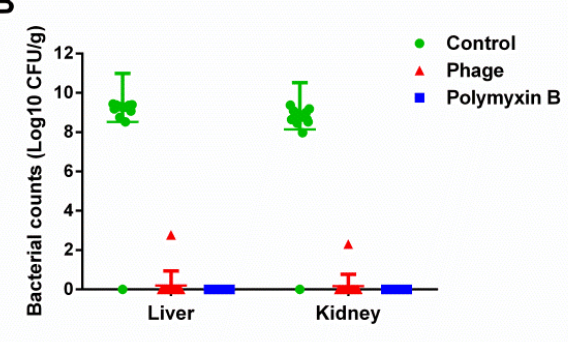

Fig. 7. Therapeutic effect of the Abp1 in systemic infection. (A) Survival of mice that were systemically infected with $\mathrm{AB} 1$ and then treated with $\mathrm{Abp} 1$ phage, polymyxin $\mathrm{B}$, or left untreated. (B) Bacterial counts in the livers and kidneys of mice that died during the observation period or were sacrificed 7 days after infection. (C) Phage titers in the livers and kidneys of each mouse. Phages or polymyxin B were injected intraperitoneally immediately after AB1 infection and treatments were given daily for 6 days. Data shown are from one of three independent experiments using 14 mice per group. ${ }^{*} \mathrm{P}<0.05$, ${ }^{*} \mathrm{P}<0.05$ compared with the negative control.

(Fig. 7B). Finally, in phage-treated animals, phage were detected in both livers and kidneys (approximately $10^{4}$ to $10^{5} \mathrm{PFU} / \mathrm{g}$ ), suggesting that phage replication occurred in vivo (Fig. 7C). Together, these data suggest that Abp1 is highly effective against systemic A. baumannii infection.

\section{Discussion}

Because of increasing drug resistance, $A$. baumannii has become a serious threat to human health [15]. Phages, as "our enemy's enemy," are potential weapons against drugresistant pathogens $[16,17]$. Some phage products have already been approved by the FDA as food biopreservatives, such as ListShield and ListexP100 [18]. The Phagoburn clinical study, supported by several European countries, was conducted to assess the safety, effectiveness, and pharmacodynamics of two therapeutic phage cocktails to treat E. coli and P. aeruginosa burn wound infections [19]. In our previous survey, we found that A. baumannii was highly prevalent in our hospital, and a majority of isolates exhibited multi-drug resistance or pan-drug resistance $[20,21]$. To explore the possibility that phage therapy might be effective against this pathogen, we isolated $21 \mathrm{~A}$. baumannii phage strains from the hospital environment. Although nearly all had narrow host ranges, Abp1 exhibited a relatively wide host range. About 5 percent of over 200 clinical A. baumannii isolates are sensitive to Abp1 (data not shown). We also tested several phage cocktails, but the cocktail host range was the simple sum of the phage component host ranges. In our future work, we want to expand the host range by two ways. Firstly, isolate more phages against $A$. baumannii strains belonging to different ST types. Secondly, explore CRISPR-Cas9 technology to manipulate the phage tails which play an important role in recognizing host receptors.

The results presented here show that Abp1 has several qualities that are desirable for a potential antimicrobial agent. Abp1 is stable under a wide range of temperatures and $\mathrm{pH}$ 
environments, and has almost no detectable toxicity against HeLa or THP-1 cells. In addition, the A. baumannii isolate we used in our work exhibits phage resistance at very low levels, and lysogeny is rare or perhaps nonexistent. Abp1 can lyse nearly all cells even at low MOI, suggesting that a small number of phage may be sufficient for effective phage therapy.

In the wound infection model, topically applied Abp1 accelerated the healing of AB1infected wounds. However, when phage are introduced systemically via subcutaneous injection, the effect is indistinguishable from the negative control. This result indicates that topical administration is a better choice for the treatment of local infections. In the systemic infection model, phage-treated animals exhibited a 100\% survival rate when phage are introduced immediately after bacterial infection. Abp1 efficacy is equivalent to that of polymyxin $B(10 \mathrm{mg} / \mathrm{kg})$, and phage therapy is therefore a potential treatment for pan-drug resistant $A$. baumannii infections.

\section{Conclusion}

We have evaluated the safety, effectiveness, and methods of administration for Abp1 in the treatment of $A$. baumannii local and systemic infections. Our results provide evidence that phage therapy can be effective against multi-drug resistant $A$. baumannii infections, and suggest that additional clinical studies are warranted to confirm and expand these findings.

\section{Disclosure Statement}

No conflict of interests exists.

\section{Acknowledgements}

We would like to thank Dr. Shouguang Jin (University of Florida, USA) for providing assistance with English grammar.

This work was supported by National Natural Science Foundation of China (No. 81571896 and No. 81772073) and Technological Innovation Plan in Major Fields of Southwest Hospital, Key Projects (No. SWH2016ZDCX2001).

\section{References}

$>1$ Howard A, O’Donoghue M, Feeney A, Sleator RD: Acinetobacter baumannii an emerging opportunistic pathogen. Virulence 2012;3:243-250.

-2 Peleg AY, Seifert H, Paterson DL: Acinetobacter baumannii: Emergence of a successful pathogen. Clin Microbiol Rev 2008;21:538-582.

3 Song CT, Hwee J, Song C, Tan BK, Chong SJ: Burns infection profile of singapore: Prevalence of multidrugresistant acinetobacter baumannii and the role of blood cultures. Burns Trauma 2016;4:13.

-4 Fournier PE, Vallenet D, Barbe V, Audic S, Ogata H, Poirel L, Richet H, Robert C, Mangenot S, Abergel C, Nordmann P, Weissenbach J, Raoult D, Claverie JM: Comparative genomics of multidrug resistance in acinetobacter baumannii. PLoS Genet 2006;2:e7.

5 Summers WC: Bacteriophage therapy. Annu Rev Microbiol 2001;55:437-451.

-6 Seth AK, Geringer MR, Nguyen KT, Agnew SP, Dumanian Z, Galiano RD, Leung KP, Mustoe TA, Hong SJ: Bacteriophage therapy for staphylococcus aureus biofilm-infected wounds: A new approach to chronic wound care. Plast Reconstr Surg 2013;131:225-234.

7 Harper DR, Morales S: Bacteriophage therapy: Practicability and clinical need meet in the multidrugresistance era. Future Microbiol 2012;7:797-799. 


\section{Cellular Physiology Cell Physiol Biochem 2017;44:2337-2345 \begin{tabular}{l|l} 
DOI: 10.1159/000486117 & $\begin{array}{l}\text { O 2017 The Author(s). Published by S. Karger AG, Basel } \\
\text { www.karger.com/cpb }\end{array}$
\end{tabular}}

Yin et al.: Phage Abp1 in Treating A. Baumannii Infections

8 Wu S, Zachary E, Wells K, Loc-Carrillo C: Phage therapy: Future inquiries. Postdoc J 2013;1:24-35.

9 Levin BR, Bull JJ: Population and evolutionary dynamics of phage therapy. Nat Rev Microbiol 2004;2:166173.

10 Mattila S, Ruotsalainen P, Jalasvuori M: On-demand isolation of bacteriophages against drug-resistant bacteria for personalized phage therapy. Front Microbiol 2015;6:1271.

11 Huang G, Le S, Peng Y, Zhao Y, Yin S, Zhang L, Yao X, Tan Y, Li M, Hu F: Characterization and genome sequencing of phage abp1, a new phikmv-like virus infecting multidrug-resistant acinetobacter baumannii. Curr Microbiol 2013;66:535-543.

12 Garrison AT, Abouelhassan Y, Kallifidas D, Bai F, Ukhanova M, Mai V, Jin S, Luesch H, Huigens RW, 3rd: Halogenated phenazines that potently eradicate biofilms, mrsa persister cells in non-biofilm cultures, and mycobacterium tuberculosis. Angew Chem Int Ed Engl 2015;54:14819-14823.

13 Thompson MG, Black CC, Pavlicek RL, Honnold CL, Wise MC, Alamneh YA, Moon JK, Kessler JL, Si Y, Williams R, Yildirim S, Kirkup BC, Jr., Green RK, Hall ER, Palys TJ, Zurawski DV: Validation of a novel murine wound model of acinetobacter baumannii infection. Antimicrob Agents Chemother 2014;58:1332-1342.

14 Huang G, Shen X, Gong Y, Dong Z, Zhao X, Shen W, Wang J, Hu F, Peng Y: Antibacterial properties of acinetobacter baumannii phage abp1 endolysin (plyab1). BMC Infect Dis 2014;14:681.

15 Giamarellou H, Antoniadou A, Kanellakopoulou K: Acinetobacter baumannii: A universal threat to public health? Int J Antimicrob Agents 2008;32:106-119.

16 Bradbury J: “My enemy's enemy is my friend." Using phages to fight bacteria. Lancet 2004;363:624-625.

17 Teng T, Liu J, Wei H: Anti-mycobacterial peptides: From human to phage. Cell Physiol Biochem 2015;35:452-466.

18 Sarhan WA, Azzazy HM: Phage approved in food, why not as a therapeutic? Expert Rev Anti Infect Ther 2015;13:91-101.

19 Matsuzaki S, Uchiyama J, Takemura-Uchiyama I, Daibata M: Perspective: The age of the phage. Nature 2014;509:S9.

20 Gong Y, Shen X, Huang G, Zhang C, Luo X, Yin S, Wang J, Hu F, Peng Y, Li M: Epidemiology and resistance features of acinetobacter baumannii isolates from the ward environment and patients in the burn icu of a chinese hospital. J Microbiol 2016;54:551-558.

21 Huang G, Yin S, Gong Y, Zhao X, Zou L, Jiang B, Dong Z, Chen Y, Chen J, Jin S, Yuan Z, Peng Y: Multilocus sequence typing analysis of carbapenem-resistant acinetobacter baumannii in a chinese burns institute. Front Microbiol 2016;7:1717. 